\title{
An Evaluation of Model Output Statistics for Subseasonal Streamflow Forecasting in European Catchments
}

\author{
SimON SCHICK, Ole RÖSSLER, AND ROLF WEINGARTNER \\ Institute of Geography, and Oeschger Centre for Climate Change Research, University of Bern, Bern, Switzerland
}

(Manuscript received 10 September 2018, in final form 10 May 2019)

\begin{abstract}
Subseasonal and seasonal forecasts of the atmosphere, oceans, sea ice, or land surfaces often rely on Earth system model (ESM) simulations. While the most recent generation of ESMs simulates runoff per land surface grid cell operationally, it does not typically simulate river streamflow directly. Here, we apply the model output statistics (MOS) method to the hindcast archive of the European Centre for Medium-Range Weather Forecasts (ECMWF). Linear models are tested that regress observed river streamflow on surface runoff, subsurface runoff, total runoff, precipitation, and surface air temperature simulated by ECMWF's forecast systems S4 and SEAS5. In addition, the pool of candidate predictors contains observed precipitation and surface air temperature preceding the date of prediction. The experiment is conducted for 16 European catchments in the period 1981-2006 and focuses on monthly average streamflow at lead times of 0 and 20 days. The results show that skill against the streamflow climatology is frequently absent and varies considerably between predictor combinations, catchments, and seasons. Using streamflow persistence as a benchmark model further deteriorates skill. This is most pronounced for a catchment that features lakes, which extend to about $14 \%$ of the catchment area. On average, however, the predictor combinations using the ESM runoff simulations tend to perform best.
\end{abstract}

\section{Introduction}

Subseasonal and seasonal forecasts of environmental conditions are increasingly based on numerically coupled models of the various Earth system components. These include general circulation models of the atmosphere and oceans and dynamical land surface or sea ice models (National Academies 2016).

Such forecast systems represent diverse physical, chemical, and biological processes and continuously progress toward Earth system models (ESMs). However, not all environmental variables of interest are resolved. For example, current generation ESMs simulate runoff per land surface grid cell operationally, but they do not typically simulate river streamflow (Clark et al. 2015; Yuan et al. 2015).

To the best of our knowledge, ESM runoff simulations have been virtually ignored for subseasonal and seasonal streamflow forecasting with the exception of the following studies:

- Yuan and Wood (2012) predict seasonal streamflow in the Ohio basin using hindcasts of the Climate Forecast System

Corresponding author: Simon Schick, simon.schick@giub.unibe.ch version 2 (CFSv2). Besides forcing the VIC hydrological model with CFSv2 climate predictions, the authors also postprocess the CFSv2 runoff simulations with a linear routing model and a statistical bias correction. The results highlight the importance of the statistical bias correction and show that the postprocessed runoff simulations provide a serious benchmark for the calibrated VIC model.

- Emerton et al. (2018) introduce the Global Flood Awareness System (GloFAS) seasonal forecasting system. This system builds upon the forecasting capabilities of the European Centre for Medium-Range Weather Forecasts (ECMWF) and feeds runoff simulated by the ESM land surface scheme to the LISFLOOD model: Subsurface runoff enters a groundwater module and streamflow is routed according to the kinematic wave equations.

A different approach to predict river streamflow with ESM-based runoff simulations exists in the application of the model output statistics (MOS) method. The MOS method emerged in the context of weather prediction (Glahn and Lowry 1972; Klein and Glahn 1974), where it regressed the variable of interest against the output of a numerical weather model. Today, the MOS method usually has to deal with an ensemble of model integrations, 
which accounts for uncertainties regarding the initial conditions and model implementation (e.g., Schefzik et al. 2013).

Broadly speaking, the MOS method attempts to statistically model the correlation of dynamical forecasts and corresponding observations. Besides the prediction of variables not resolved by the dynamical model, the MOS method also can target bias correction (Barnston and Tippett 2017), model combination (Slater et al. 2017), and the modeling of the forecast's probability distribution (Zhao et al. 2017). Often, several of these targets are addressed at the same time.

The MOS method is also sporadically used to predict river streamflow at the subseasonal and seasonal time scales. Early examples include Landman and Goddard (2002) and Foster and Uvo (2010), while more recently the approaches of Sahu et al. (2017), Lehner et al. (2017), or Slater and Villarini (2018) fall within the realm of the MOS method.

In most of these studies, the predictand consists of (sub) seasonal streamflow volumes and the model formulation is based on the assumption of linear predictor-predictand relationships. However, the selected predictors vary considerably and include ESM-simulated precipitation, wind velocity, surface air temperature, the geopotential height of atmospheric pressure levels, or time series of land use cover and population density.

Here, we test the application of the MOS method to ESM-based subseasonal forecasts of surface, subsurface, and total runoff. In addition, models are formulated that include precipitation and surface air temperature as predictors. The present implementation of the MOS method relies on the linear regression model and is prototyped in Schick et al. (2018). To mature the prototype we add an error model and conduct a validation in 16 European river systems featuring a range of climatic and geographical conditions.

The hindcast experiment uses data from both the former (S4) as well as current (SEAS5, or S5 in short) seasonal forecast systems of ECMWF. To separate the skill originating from the traditional weather forecasting time scale and the potential skill at the subseasonal time scale, the predictand is defined as mean streamflow of a time window of 30 days with lead times of 0 and 20 days.

Below, section 2 introduces the dataset, section 3 details the MOS method and the hindcast verification, sections 4 and 5 present and discuss the results, and section 6 concludes the study.

\section{Data}

The hydrometeorological data cover the time period 19812006 and have a daily temporal resolution. Spatial fields get aggregated by taking catchment area averages based on the percent of grid cell coverage of the catchment polygon. In addition, each grid cell is weighted by the cosine of its latitude to account for the meridional variation of the grid cell area.

\section{a. Catchments}

Table 1 and Fig. 1 show the set of the selected 16 catchments, which includes lowlands and mountainous regions as well as subarctic, temperate, mediterranean, and humid-continental climate types (Peel et al. 2007; Mücher et al. 2010). The catchment areas approximately range from 5000 to $285600 \mathrm{~km}^{2}$.

These river systems are subject to damming and streamflow regulation of varying degree (Nilsson et al. 2005). However, human activities affecting river streamflow can hardly be avoided when it comes to streamflow forecasting in large European river systems. Whether these human activities lead to a pattern that can be learned by the MOS method or instead are a source of noise will be discussed later in more detail.

\section{b. Observations}

Daily mean streamflow observations $\left(\mathrm{m}^{3} \mathrm{~s}^{-1}\right)$ are provided by the Global Runoff Data Centre (GRDC 2016), the Spanish Ministry of Agriculture and Fisheries, Food and Environment (MAFFE 2017), and the French Ministry for an Ecological and Solidary Transition (MEST 2017). Catchment polygons are either retrieved from the GRDC (2016) or derived from the European catchments and Rivers network system (ECRINS 2012).

Daily observations of surface air temperature $\left({ }^{\circ} \mathrm{C}\right)$ and precipitation $(\mathrm{mm})$ are taken from the ENSEMBLES gridded observational dataset in Europe, version 16.0 (EOBS). This dataset is based on a statistical interpolation of weather station observations and is available on a $0.25^{\circ}$ regular grid (Haylock et al. 2008; E-OBS 2017).

\section{c. Hindcast archive}

ECMWF's former (S4) and current (S5) seasonal forecast systems consist of numerically coupled atmosphereocean-land models; in addition, S5 includes the LIM2 sea ice model. The net horizontal resolution of the atmosphere model equals about $80 \mathrm{~km}$ (S4) and $35 \mathrm{~km}$ (S5), whereas the NEMO ocean model approximately operates on a $1^{\circ}$ (S4) and $0.25^{\circ}$ (S5) grid (ECMWF 2017).

The HTESSEL land surface model, which is part of both S4 and S5, dynamically divides each grid cell into fractions of bare ground, low and high vegetation, intercepted water, snow, and snow under high vegetation. The partitioning into infiltration and surface runoff happens according to the Arno scheme. Vertical water flow in the soil, which is discretized into four layers with a total depth of about $3 \mathrm{~m}$, follows the Richards equation. Total runoff finally equals the sum of surface runoff and open drainage at the soil bottom (Balsamo et al. 2009; ECMWF 2018). 
TABLE 1. Selected catchments and corresponding sites of gauging stations, data providers, catchment areas, and average streamflows in the period 1981-2006.

\begin{tabular}{|c|c|c|c|c|c|}
\hline & Name & Site & Provider & Catchment area $\left(\mathrm{km}^{2}\right)$ & Average streamflow $\left(\mathrm{m}^{3} \mathrm{~s}^{-1}\right)$ \\
\hline 1 & Angerman & Sollefteå & GRDC & 30900 & 532 \\
\hline 2 & Danube & Bratislava & GRDC & 131400 & 2072 \\
\hline 3 & Duero & Herrera de Duero & MAFFE & 12900 & 24 \\
\hline 4 & Elbe & Neu-Darchau & GRDC & 131100 & 691 \\
\hline 5 & Garonne & Lamagistère & MEST & 32300 & 386 \\
\hline 6 & Glama & Langnes & GRDC & 39900 & 702 \\
\hline 7 & Kemijoki & Isohaara & GRDC & 53900 & 577 \\
\hline 8 & Lower Bann & Movanagher & GRDC & 5000 & 92 \\
\hline 9 & Neva & Novosaratovka & GRDC & 285600 & 2479 \\
\hline 10 & Oder & Hohensaaten-Finow & GRDC & 110500 & 492 \\
\hline 11 & Rhine & Lobith & GRDC & 159400 & 2335 \\
\hline 12 & Rhone & Beaucaire & MEST & 95800 & 1707 \\
\hline 13 & Seine & Paris & MEST & 43600 & 315 \\
\hline 14 & Tisza & Senta & GRDC & 141100 & 795 \\
\hline 15 & Torne & Kukkolankoski & GRDC & 40500 & 423 \\
\hline 16 & Trent & Colwick & GRDC & 7500 & 84 \\
\hline
\end{tabular}

For both S4 and S5 the hindcast spans back to 1981 with initial conditions taken from ERA-Interim. Reforecasts are initialized on the first day of each month and simulate the subsequent 7 months. The number of hindcast ensemble members equals 15 (S4) and 25 (S5), respectively. Please note that this describes ECMWF's standard hindcast configuration, that is, for certain dates of prediction more ensemble members and a longer lead time are available.

We downloaded the following variables on a regular $0.75^{\circ}(\mathrm{S} 4)$ and $0.4^{\circ}(\mathrm{S} 5)$ grid at a daily resolution: accumulated precipitation $(\mathrm{m})$, air temperature $2 \mathrm{~m}$ above ground $(\mathrm{K})$, accumulated total runoff, surface runoff, and subsurface runoff $(\mathrm{m})$. Surface and subsurface runoff is only available for the S5 system.

After taking catchment area averages as described above, accumulated variables are first converted to daily fluxes; in addition, total runoff, surface runoff, and subsurface runoff are converted from meters $(\mathrm{m})$ to meters cubed per second $\left(\mathrm{m}^{3} \mathrm{~s}^{-1}\right)$ and surface air temperature is converted from kelvins $(\mathrm{K})$ to degrees Celsius $\left({ }^{\circ} \mathrm{C}\right)$. Finally, the ensemble gets compressed to its mean value.

\section{Method}

The predictand $y_{w, l}$ denotes mean streamflow $\left(\mathrm{m}^{3} \mathrm{~s}^{-1}\right)$ of a time window with length $w=30$ days and lead time $l=0,20$ days. Here, lead time is defined as the time difference between the date of prediction and the onset of the actual prediction window $w$. The date of prediction is set to the first day of the month in the period 1981-2006.

To predict with a 20-day lead time, we do not regress $y_{30,20}$, but instead predict $\hat{y}_{20,0}$ and $\hat{y}_{50,0}$, followed by integration in time and taking differences, that is,

$$
\hat{y}_{30,20}=\left(\hat{y}_{50,0} \times 50-\hat{y}_{20,0} \times 20\right) / 30 .
$$

Doing so allows us to center the model formulation around the date of prediction without the need to account for the temporal gap introduced by the lead time $l$ (section 3a).

Thus, for the regression we effectively use $w=30,30,50$ days and $l=0$ days. Furthermore, the modeling procedure is individually applied for each prediction window $w$ and

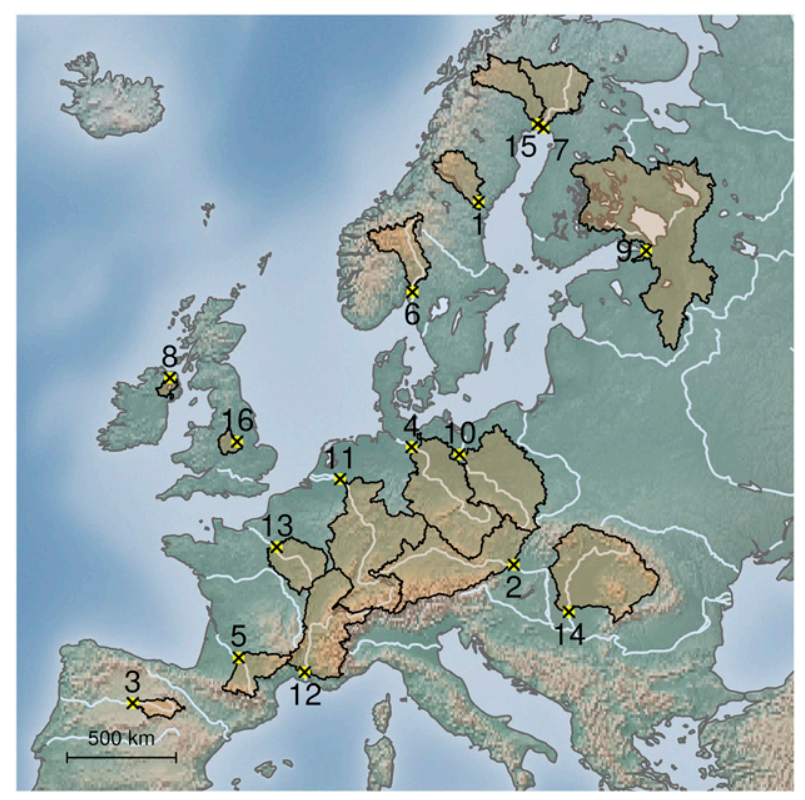

FIG. 1. The hindcast experiment is conducted for 16 catchments situated in Europe. Black crosses on a yellow background indicate the sites of the gauging stations, light blue lines show some large rivers, and the numbers refer to the entries in Table 1 . The map is produced with data from Natural Earth (2018). 
TABLE 2. The predictor combinations consider the variables $p$ : precipitation, $t$ : surface air temperature, ro: total runoff, sro: surface runoff, and ssro: subsurface runoff. Predictors get aggregated in time either preceding or subsequent to the date of prediction; the subscripts indicate the data source, i.e., the E-OBS dataset and the S4 and S5 hindcast archives. Predictors derived from the S4 and S5 archives are based on the ensemble mean.

\begin{tabular}{|c|c|c|c|c|c|}
\hline \multirow[b]{2}{*}{ Name } & \multicolumn{2}{|c|}{ Preceding } & \multicolumn{2}{|c|}{ Subsequent } & \multirow[b]{2}{*}{ Description } \\
\hline & $x_{1}$ & $x_{2}$ & $x_{3}$ & $x_{4}$ & \\
\hline refRun & $p_{\mathrm{E}-\mathrm{OBS}}$ & $t_{\mathrm{E}-\mathrm{OBS}}$ & $p_{\mathrm{E}-\mathrm{OBS}}$ & $t_{\mathrm{E}-\mathrm{OBS}}$ & Precipitation and temperature from the E-OBS dataset \\
\hline preMet & $p_{\mathrm{E}-\mathrm{OBS}}$ & $t_{\mathrm{E}-\mathrm{OBS}}$ & - & - & Precipitation and temperature from the E-OBS dataset \\
\hline S4PT & $p_{\text {E-OBS }}$ & $t_{\mathrm{E}-\mathrm{OBS}}$ & $p_{\mathrm{S} 4}$ & $t_{\mathrm{S} 4}$ & $\begin{array}{l}\text { Precipitation and temperature from the E-OBS dataset } \\
\text { and S4 archive }\end{array}$ \\
\hline S5PT & $p_{\mathrm{E}-\mathrm{OBS}}$ & $t_{\mathrm{E}-\mathrm{OBS}}$ & $p_{\mathrm{S} 5}$ & $t_{\mathrm{S} 5}$ & $\begin{array}{l}\text { Precipitation and temperature from the E-OBS dataset } \\
\text { and S5 archive }\end{array}$ \\
\hline S4ro & - & - & $\mathrm{ro}_{\mathrm{S} 4}$ & - & Total runoff from the $\mathrm{S} 4$ archive \\
\hline S5ro & - & - & $\operatorname{ros}_{5}$ & - & Total runoff from the $\mathrm{S} 5$ archive \\
\hline S5sro+ssro & - & - & $\operatorname{sro}_{S 5}$ & ssros5 $_{5}$ & Surface and subsurface runoff from the S5 archive \\
\hline
\end{tabular}

date of prediction within the calendar year, leaving 26 years to perform the regression. Having said this, we drop for the following the subscripts $w$ and $l$.

\section{a. Regression model}

\section{1) Time aggregation SCREening}

The time aggregation of a particular predictor is defined with respect to the date of prediction and involves summation (precipitation) or averaging (surface air temperature and runoff) in time. The time aggregation period is not fixed in advance, but is individually selected for each predictor based on the linear correlation with $y$. It is constrained to the sets

- $A_{\text {pre }}=\{10,20, \ldots, 720\}$ days for predictors that carry information preceding the date of prediction (backward in time; columns $x_{1}$ and $x_{2}$ in Table 2), and

- $A_{\text {sub }}=\{5,10, \ldots, 200\}$ days for predictors that carry information subsequent to the date of prediction (forward in time; columns $x_{3}$ and $x_{4}$ in Table 2). For the refRun model (introduced below) we set $A_{\text {sub }}=\{5,10, \ldots, w+l\}$ days.

In so doing the time window of the ESM-based predictors can differ from the actual forecast window. This allows us to account for a delayed catchment response to the atmospheric forcings or could help to better detect skillfully predicted climate anomalies.

\section{2) Predictor combinations}

The regression equation is given by

$$
y=\psi(\mathbf{x}, D)+\varepsilon=\mathbf{x}^{\mathrm{T}} \hat{\boldsymbol{\beta}}+\varepsilon,
$$

with $\mathbf{x}^{\mathrm{T}}=\left[1 x_{1} x_{2} x_{3} x_{4}\right]$ being the predictor vector and $\boldsymbol{\beta}$ the coefficient vector. Both the time aggregation periods of the entries in $\mathbf{x}$ as well as the ordinary least squares estimate of $\boldsymbol{\beta}$ are based on the training set $D$. Please note that we do not make any distributional assumption about the error term $\varepsilon$.

Table 2 shows the different predictor combinations that make up x. The models named refRun (reference run) and preMet (preceding meteorology) are intended to provide an upper and lower boundary of prediction skill when using precipitation and surface air temperature as predictors: For the refRun model we plug in observed precipitation and surface air temperature preceding the date of prediction (i.e., a proxy for the initial hydrological conditions) as well as observed precipitation and surface air temperature subsequent to the date of prediction (i.e., assuming perfect seasonal climate predictions). In contrast, the preMet model does not have any information about the climate of the target period.

The remaining models contain predictors from the $\mathrm{S} 4$ and S5 hindcast archives, which all base on the ensemble mean: besides precipitation and surface air temperature, we test total runoff as well as surface and subsurface runoff as individual predictors. Please see appendix A for a technical note concerning the S5sro+ssro model.

\section{3) Bootstrap AgGREGATING}

Bootstrap aggregating (bagging) dates back to Breiman (1996a) and is a technique to reduce model variance. For the present prediction problem and modeling strategy, bagging helps to stabilize model variance as introduced by the small sample size and the sometimes weak relationships (Schick et al. 2016). The bagged prediction follows

$$
\hat{y}=\frac{1}{b} \sum_{j=1}^{b} \psi\left(\mathbf{x}, D_{j}\right)
$$

where the subscript $j$ indicates the $j$ th nonparametric bootstrap replicate of $D$. Please note that the number of 
bootstrap replicates $b$ should not be regarded as a tuning parameter, but is set to a value such that the prediction error stabilizes. To guarantee the robustness of the analysis we set $b=100$, which can be considered as rather high [e.g., Breiman (1996a) recommends $b \in\{25, \ldots, 50\}]$.

\section{b. Error model}

The error model employs the so-called "out-of-bag" prediction error estimate (Breiman 1996b), which avoids an additional cross validation. In each of the $b$ bootstrap replicates we (most likely) miss some of the cases contained in the full training set. Thus, for the $i$ th case $\left(y_{i}, \mathbf{x}_{i}\right) \in D$ we can approximate its prediction error according to

$$
\hat{\varepsilon}_{i}=y_{i}-\frac{1}{\sum_{j=1}^{b} 1\left(y_{i} \notin D_{j}\right)} \sum_{j=1}^{b} \psi\left(\mathbf{x}_{i}, D_{j}\right) \times 1\left(y_{i} \notin D_{j}\right),
$$

with $1(\cdot)$ denoting the indicator function that returns one if its argument evaluates to true and zero otherwise. Here, the indicator function excludes those models from the model averaging in Eq. (3) that use $\left(y_{i}, \mathbf{x}_{i}\right)$ for the time aggregation screening and estimation of $\boldsymbol{\beta}$. For the 20-day lead time, Eq. (4) needs to be adapted according to Eq. (1).

Having estimated the prediction error for each case in the training set, we then use a kernel density estimate to specify the probability density function $f$ of a future prediction $\hat{y}$

$$
\hat{f}(y)=\frac{1}{n h} \sum_{i=1}^{n} K\left(\frac{y-\hat{y}-\hat{\varepsilon}_{i}}{h}\right),
$$

with $n$ being the sample size of the training set $D$ and the kernel $K(z)$ the standard Gaussian density function

$$
K(z)=\frac{1}{\sqrt{2 \pi}} \exp \left(-\frac{1}{2} z^{2}\right) .
$$

The bandwidth parameter $h>0$ is automatically selected according to the method of Sheather and Jones (1991) as implemented in the statistical software R (R Core Team 2018). This method belongs to the "solvethe-equation plug-in" approaches and relies on the minimization of the asymptotic mean integrated squared error (AMISE). The method seems to work well for a variety of density shapes as it uses a bandwidth independent of $h$ to estimate the second derivative of the unknown density function in the AMISE (Jones et al. 1996).

\section{c. Verification}

The modeling procedure outlined in sections $3 \mathrm{a}$ and $3 b$ is subject to a buffered leave-one-out scheme. A buffer of 2 years to the right and left of the left-out year is used in order to avoid artificial skill due to hydrometeorological persistence (Michaelsen 1987).

The persistence benchmark model [section $3 \mathrm{c}(5)$ below] requires streamflow observations preceding the date of prediction. Since we do not have streamflow observations prior to January 1981, we miss the predictions of the persistence model in January 1981. Thus, we decide to exclude January 1981 for the entire verification.

\section{1) Reliability}

Reliable forecast distributions reproduce the observations' frequency, that is, the forecast distribution is neither too narrow (overconfident) nor too wide (underconfident). Here, we follow Laio and Tamea (2007), who propose to evaluate the probability integral transform (PIT) values graphically via the empirical cumulative distribution function. The PIT value of a forecasted cumulative distribution function $\hat{F}_{i}(y)$ and corresponding observation $y_{i}$ is defined as the probability

$$
\mathrm{PIT}=\hat{F}_{i}\left(y_{i}\right) .
$$

If $y$ is continuous and the forecasts are reliable, the PIT values follow the uniform distribution $U(0,1)$.

\section{2) AsSOCIATION AND ACCURACY}

The following scoring rules are employed: the linear correlation coefficient of the predictions and observations, the mean absolute error (MAE), the mean squared error (MSE), and the continuous ranked probability score (CRPS). The CRPS, averaged over $n$ cases in the hindcast period, is defined as (Hersbach 2000)

$$
\overline{\mathrm{CRPS}}=\frac{1}{n} \sum_{i=1}^{n} \int_{-\infty}^{\infty}\left[\hat{F}_{i}(y)-H\left(y-y_{i}\right)\right]^{2} d y,
$$

with $H(\cdot)$ denoting the Heaviside function

$$
H(x)= \begin{cases}0, & x<0 \\ 1, & x \geq 0 .\end{cases}
$$

\section{3) SKILL}

Having a model of interest $\mathrm{m} 1$ and benchmark model $\mathrm{m} 2$, the mean absolute error skill score (MAESS) is then defined as

$$
\text { MAESS }=1-\frac{\mathrm{MAE}^{\mathrm{m} 1}}{\mathrm{MAE}^{\mathrm{m} 2}}
$$

The mean squared error skill score (MSESS) and the continuous ranked probability skill score (CRPSS) are defined analogously. 


\section{4) Statistical significAnCE}

Statistical tests are conducted conditional on the date of prediction within the calendar year (thus $n=26$ ):

- To test the PIT values for uniformity, we report the number of null hypothesis rejections of the Pearson's chi-squared test using four, five, and six equally sized bins. In addition, we use confidence bands based on the Kolmogorov-Smirnov test. In both cases the null hypothesis assumes a uniform distribution, so we set the significance level to 0.25 in order to have more control on the type II error (that is not rejecting the null hypothesis when it is in fact false). The value of the Kolmogorov-Smirnov test statistic at the 0.25 level is taken from D'Agostino and Stephens (1986).

- To test whether a model $\mathrm{m} 1$ and a benchmark $\mathrm{m} 2$ differ in terms of the MSE and $\overline{\mathrm{CRPS}}$, we use paired differences of the individual squared errors and CRPS values. The null hypothesis "the mean difference equals zero" is then tested with the two-sided $t$ test. It must be noted that the paired differences do not always follow a Gaussian distribution. However, a comparison with a nonparametric bootstrap and the Wilcoxon test showed that the $t$ test leads to the most conservative results, for that reason we only report the $p$ values of the $t$ test.

\section{5) Additional models}

To help in the interpretation of the forecast quality and to benchmark the MOS method, the following additional models are used:

- climatology - the average of the predictand in the period 1981-2006;

- trend-a linear trend model for the predictand;

- persistence-a linear regression of the predictand against observed mean streamflow of the 30 days preceding the date of prediction;

- S4ro.lm, S5ro.lm, and S5sro+ssro.lm-a linear regression of the predictand against the runoff simulations of the same time window (lm stands for linear model).

The S4ro.lm, S5ro.lm, and S5sro+ssro.lm models can be considered as simpler versions of their counterparts in Table 2 in that they neither employ Eq. (1) nor the time aggregation screening and bagging; this resembles the approach of Balsamo et al. (2015) to verify the ERAInterim/Land simulation with respect to river streamflow observations.

Identical to the models of Table 2, the above listed models condition on the date of prediction within the calendar year, they undergo the cross validation, and the forecast distribution is based on the kernel density estimate from Eq. (5). However, the residuals $\varepsilon_{i}$ are the in-sample prediction errors of the training set.

\section{Results}

To get an overview we first calculate the correlation, MAESS, and MSESS using the complete time series of observations and predictions. Based on this overview, we decide at which models to look in more detail. This is subsequently done by verifying the hindcast conditional on the date of prediction within the calendar year, validating the reliability of the error model, and conducting a probabilistic verification in terms of the CRPS.

Below, we frequently switch between scoring rules in a seemingly unmotivated fashion. However, this helps us in section $5 \mathrm{f}$ to put the hindcast results into context with other studies. In addition, the usage of both deterministic and probabilistic scoring rules enables to validate the regression model (section 3a) separately from the error model (section $3 b$ ).

\section{a. Overview}

Figure 2 shows per catchment and model the linear correlation coefficient of the predictions and corresponding observations as well as the MAESS and MSESS with the climatology as benchmark. In general, we can identify four groups of models with a distinct performance:

- The correlation of the climatology can go up to about 0.8 with the median being around 0.5 , showing that several streamflow time series exhibit a pronounced periodic component. The trend model does not show any improvement over the climatology, which consequently manifests in a MAESS and MSESS around zero.

- The persistence model shows a marked improvement over the climatology and the trend model; it performs often on a level close to the preMet model and the models using the S4 and S5 simulations. This model tends to have the largest performance variability, in particular for the MSESS, where the positive outlier belongs to the Neva and the negative outlier to the Duero catchment. On average, the persistence model reduces the MAE of the climatology by about $18 \%$ (i.e., MAESS of 0.18) and the MSE of the climatology by about $23 \%$ (i.e., MSESS of 0.23 ) at the 0 -day lead time. At the 20-day lead time, the corresponding reductions amount to $8 \%$ (MAE) and $4 \%$ (MSE).

- The preMet model and the models that use the S4 and S5 simulations often end up with a similar performance. However, it seems that the runoff-based models score best. On average, the models in this group reduce the MAE of the climatology by about $25 \%$ and the MSE of the climatology by about $40 \%$ at the 0-day lead time. At the 20-day lead time, the 

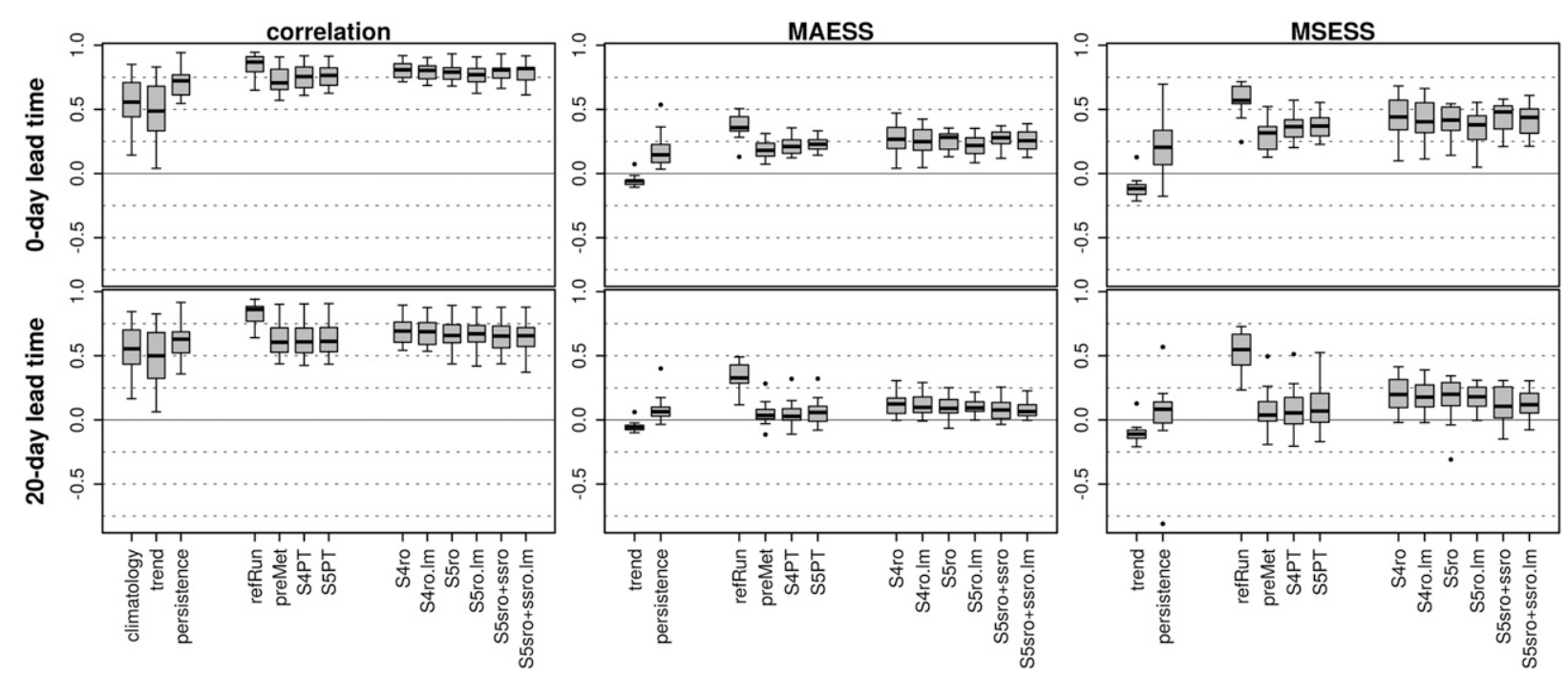

FIG. 2. Linear correlation, MAESS, and MSESS per model and catchment, based on the complete time series of predictions and observations. Shown are the (top) 0-day lead time and (bottom) 20-day lead time. The MAESS and MSESS are computed with respect to the streamflow climatology; $n=16$.

corresponding reductions amount to $9 \%$ (MAE) and $15 \%$ (MSE).

- The refRun model scores on average a correlation of about 0.85 and decreases the MAE of the climatology by about $35 \%$ and the MSE of the climatology by about $55 \%$.

For the following, we take a closer look at the S4PT, S5PT, S4ro, and S5ro models: the S4PT and S5PT models are retained as they do not use the runoff simulations. The S4ro and S5ro models are used to represent the runoff-based models, which all perform on a similar level; however, for the S5ro model we observe a negative MSESS outlier at the 20-day lead time, which could be interesting to investigate. In addition, the climatology, trend, persistence, preMet, and refRun models are retained for interpretation and benchmarking.

\section{b. Linear correlation}

The correlation coefficient per date of prediction within the calendar year, pooled to seasons, is shown in

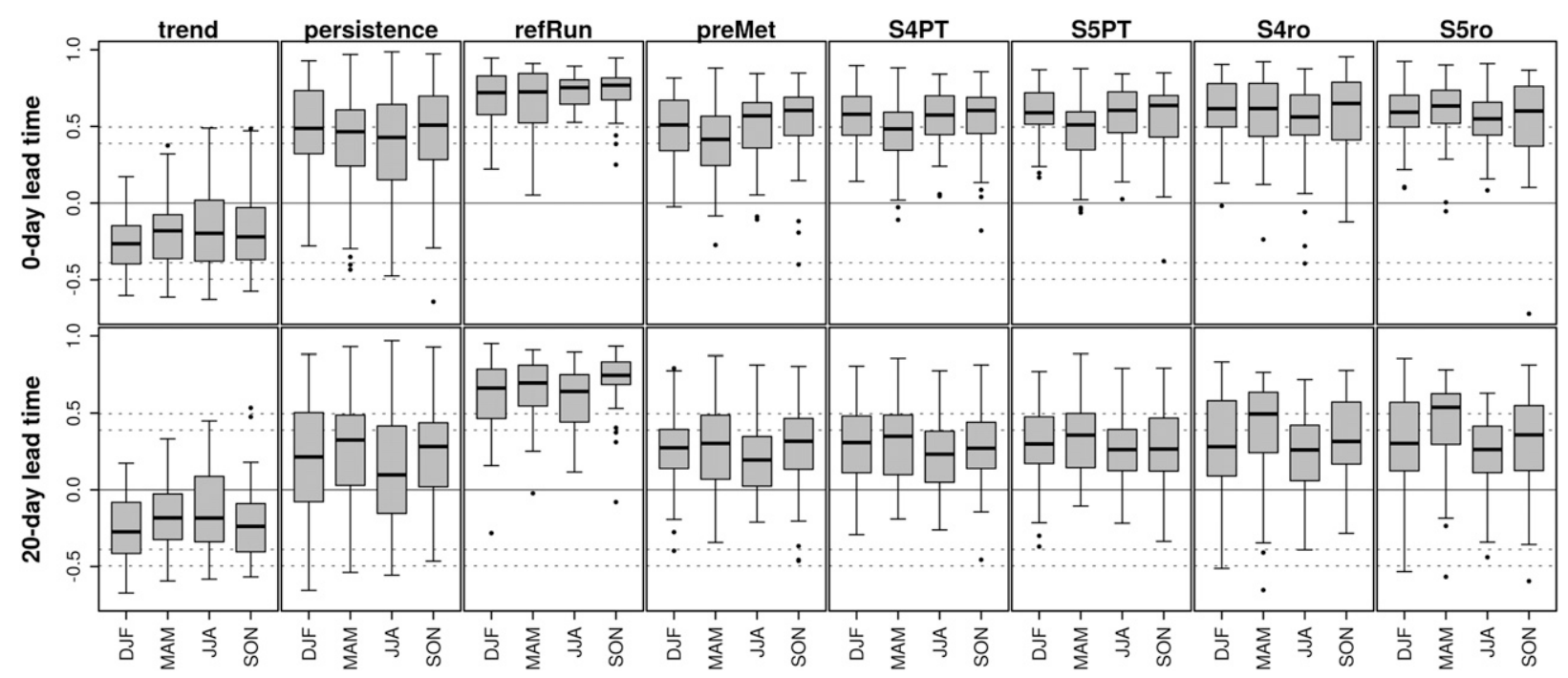

FIG. 3. Linear correlation between predictions and observations for each catchment and date of prediction within the calendar year, pooled to seasons. The dashed lines indicate the confidence intervals under the null hypothesis of zero correlation at the 0.05 and 0.01 significance level ( $t$ test for the correlation coefficient of a bivariate, normally distributed sample). Shown are the (top) 0 -day lead time and (bottom) 20-day lead time; $n=48$. 


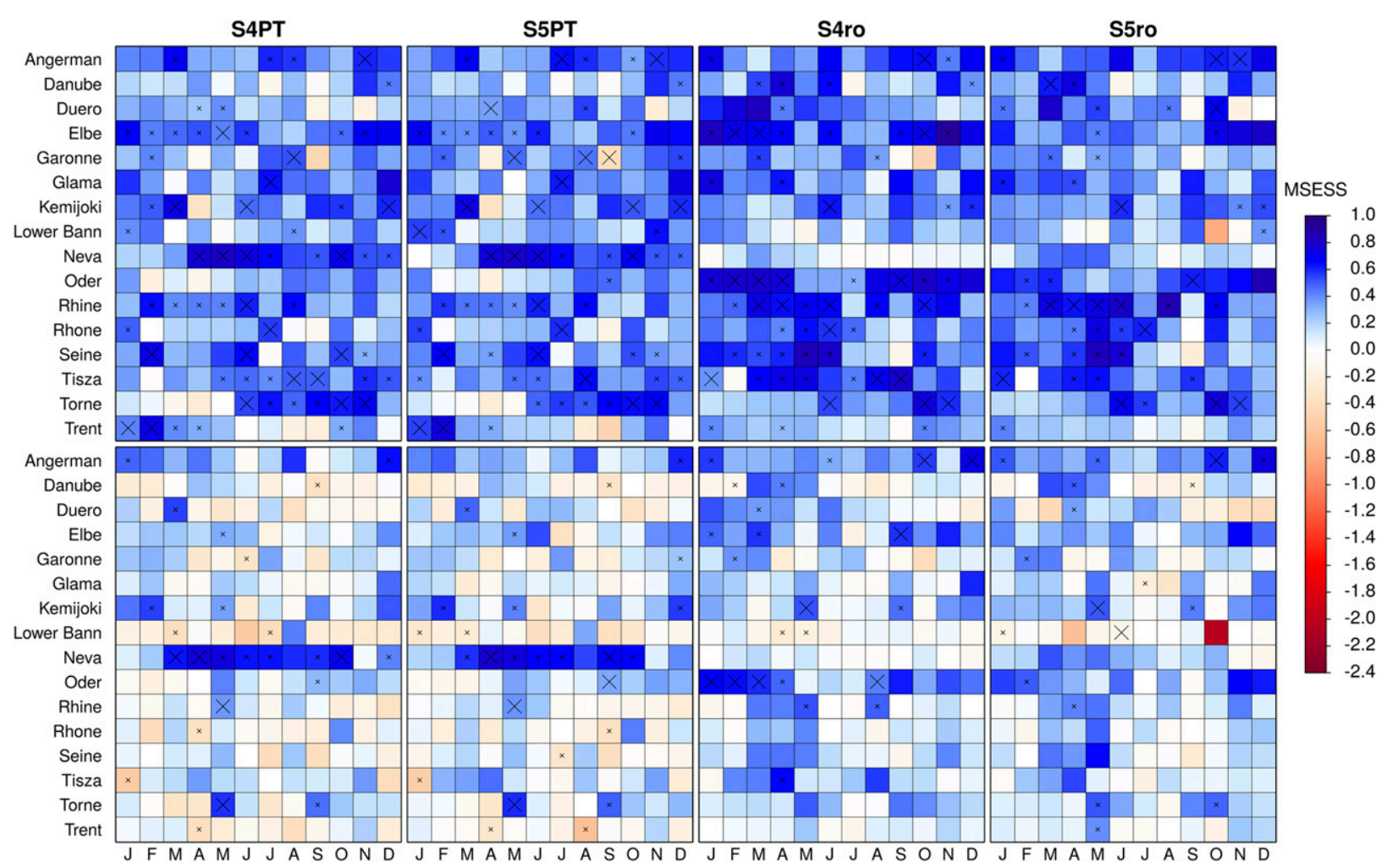

FIG. 4. MSESS at the (top) 0-day and (bottom) 20-day lead time with the streamflow climatology as the benchmark. The months refer to the date of prediction. The $p$ values smaller than 0.01 (0.05) for the null hypothesis "no difference in the MSE value" are indicated with a large (small) cross; $n=26$.

Fig. 3. The dashed lines indicate the statistical significance at the 0.05 and 0.01 levels under the null hypothesis of zero correlation ( $t$ test for the correlation coefficient of a bivariate, normally distributed sample).

In general, we observe only little seasonal variation both at the 0-day as well as the 20-day lead time, but instead a large within-season variability. Aside from the trend and refRun models, the correlation varies around 0.5 at the 0-day lead time and around 0.25 at the 20-day lead time, the latter value no longer being statistically significant. For the trend model, the correlation is mostly negative, whereas the refRun model scores a correlation around 0.7 .

\section{c. MSESS}

Figures 4 and 5 show the MSESS conditional on the date of prediction within the calendar year with the climatology as benchmark. If the paired differences in the MSE can be assumed to differ from zero according to the $t$ test, a large (small) cross is drawn in the case of the $0.01(0.05)$ significance level. The top rows correspond to the 0 -day lead time and the bottom rows to the 20-day lead time.

For the models using the S4 and S5 simulations (Fig. 4), we observe in most cases positive skill at the 0-day lead time, however, the statistical significance is frequently absent. Significant positive skill tends to cluster in spring, though a clear overall pattern does not emerge. Instead skill varies between catchments, dates of prediction, and models. At the 20-day lead time skill gets drastically reduced. Exceptions are the Oder, where the S4ro and S5ro models are not much degraded compared to the 0-day lead time, and the Neva, for which the S4PT and S5PT models still score positive skill. Here, it is also visible that the negative outlier produced by the S5ro model in Fig. 2 belongs to the Lower Bann.

The Oder and Neva show some further features (Figs. 4 and 5):

- For the Oder, we observe that the S4ro and S5ro models perform well. On the other hand, the models using the meteorological predictors, including the refRun model, perform poorly.

- The Neva seems to be the only catchment in which a linear trend contributes to skill against climatology in several months. Furthermore, the persistence, preMet, S4PT, and S5PT models score above average, while the S4ro and S5ro models instead show almost no skill. 


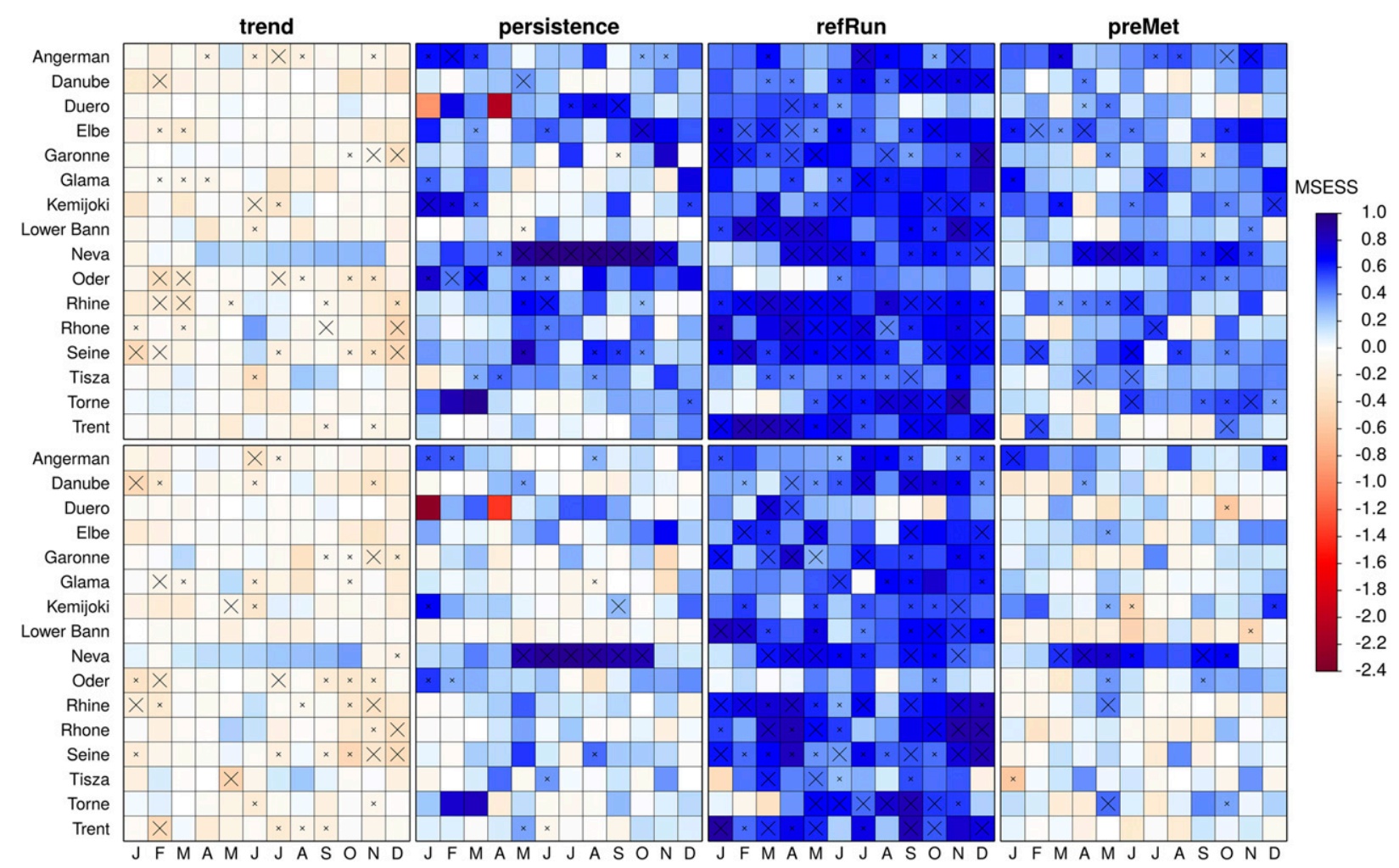

FIG. 5. MSESS at the (top) 0-day and (bottom) 20-day lead time with the streamflow climatology as the benchmark. The months refer to the date of prediction. The $p$ values smaller than $0.01(0.05)$ for the null hypothesis "no difference in the MSE value" are indicated with a large (small) cross; $n=26$.

\section{d. Reliability}

Figure 6 shows the PIT values for those models that get verified in terms of the CRPS in the next section. Shown are the empirical cumulative distribution functions of the PIT values at the 0-day lead time for each date of prediction within the calendar year, but pooled to seasons.

The distributions are accompanied by the Kolmogorov confidence bands at the 0.25 significance level. The numbers in the top-left corner report the number of rejected null hypotheses of the chisquared test based on four, five, and six bins, again at the 0.25 level. The histograms at the bottom finally pool all PIT values across seasons and catchments.

In general, we observe that the PIT distributions of the S4ro and S5ro models tend to better align with the 1: 1 diagonal than the PIT distributions of the preMet, S4PT, and S5PT models. Concerning the statistical significance, the PIT values are almost never outside the Kolmogorov confidence band. The chi-squared test rejects on average about four out of 48 distributions from being reliable.
Persistent departures from uniformity are more clear in the histograms at the bottom of Fig. 6. For all models we observe a trend toward underconfidence, that is, the tails of the forecast distributions are too heavy. For the 20-day lead time (not shown), the overall picture remains the same.

\section{e. CRPSS}

Figure 7 is similar to Figs. 4 and 5, but employs the CRPSS with the preMet model as the benchmark. Thus, for the S4PT and S5PT models skill solely originates from the S4 and S5-predicted precipitation and surface air temperature.

Starting with the 0-day lead time (top row), we observe for the S4PT and S5PT models some positive skill scattered among the catchments and dates of prediction. The S4ro and S5ro models in general do a better job, which is most evident for the Oder. On the other hand, these models score some large negative CRPSS values.

At the 20-day lead time (bottom row), skill of the S4PT and S5PT models virtually drops to zero, while the S4ro and S5ro models are still able to outperform the preMet model, most notably in the case of the Oder. 


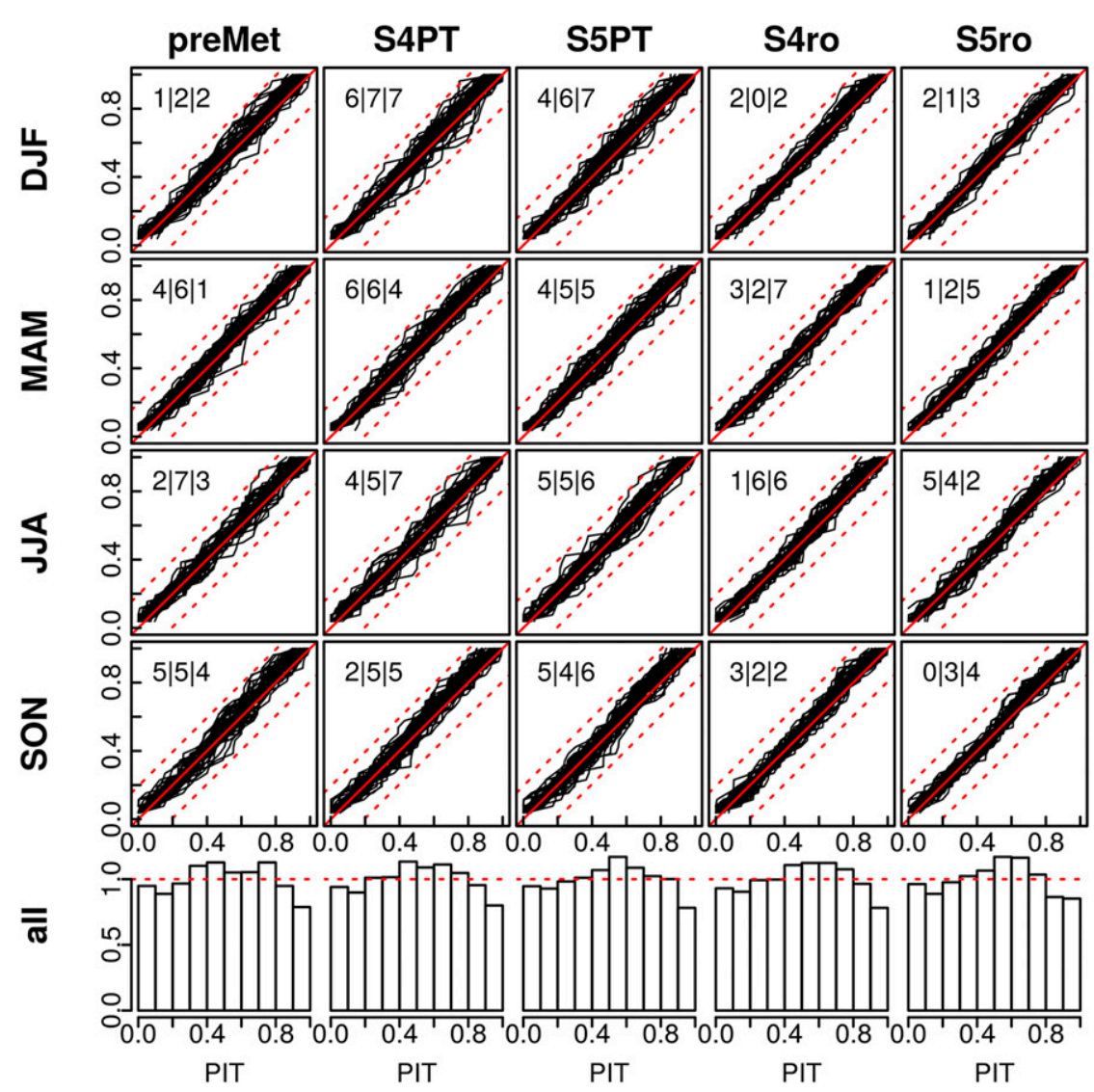

FIG. 6. Empirical cumulative distribution of the PIT values obtained at the 0-day lead time. The distribution is individually plotted for each catchment and date of prediction within the calendar year $(n=26)$ but pooled to seasons. The number of null hypothesis rejections of the chi-squared test are reported in the top left corner (corresponding to four, five, and six bins at the 0.25 significance level); the dashed red lines indicate the Kolmogorov 0.25 confidence band. The histograms at the bottom pool all PIT values across seasons and catchments $[n=(26 \times 12-1) \times 16=4976]$.

\section{f. Persistence model}

The results frequently indicate that the persistence benchmark model is challenging to beat in several catchments. Thus, appendix B contains Figs. 2, 4, 5, and 7 using the persistence model as the benchmark in the calculation of the MAESS, MSESS, and CRPSS (see Figs. B1-B4). The main results are as follows:

- One strong negative outlier is present for almost all models (see Fig. B1). This outlier with MAESS and MSESS values in the range from -0.5 to -2 belongs to the Neva. Otherwise, the MAESS scatters around 0.1 (0-day lead time) and 0.0 (20-day lead time), and the MSESS scatters around 0.25 (0-day lead time) and 0.1 (20-day lead time).

- The Neva catchment stands out as well in the other figures in appendix B (see Figs. B2, B3, and B4). MSESS values range down to -44 and CRPSS values range down to -5.5 . Otherwise, positive skill is either absent (in particular at the 20-day lead time) or does not follow an easy to interpret pattern.

\section{Discussion}

First, we discuss the validity of the regression and error models from a technical point of view. Second, we contrast the different predictor combinations. Third, we discuss the role of anthropogenic signals in the streamflow time series and potential sources of forecast skill. Finally, we compare the present hindcast results with results reported in other studies and gather the pros and cons of the MOS method.

\section{a. Regression model}

\section{1) Time aggregation}

The MOS method aims at modeling the correlation between dynamical forecasts and observations of the 


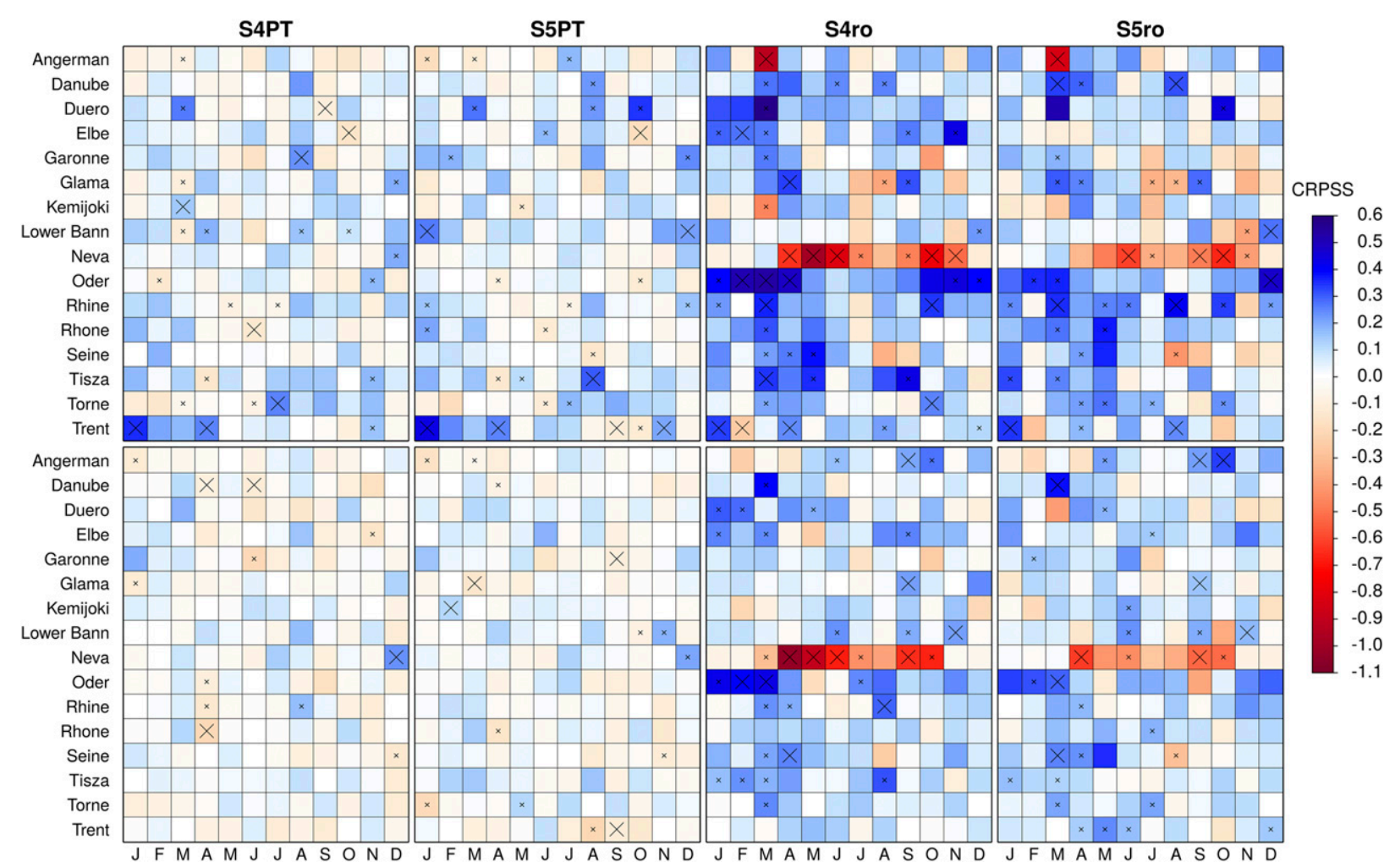

FIG. 7. CRPSS at the (top) 0-day and (bottom) 20-day lead time with the preMet model as the benchmark. The months refer to the date of prediction. The $p$ values smaller than $0.01(0.05)$ for the null hypothesis "no difference in the mean CRPS value" are indicated with a large (small) cross; $n=26$.

target variable. Apart from long-term trends and seasonal patterns, this correlation emerges at the (sub) seasonal time scale only at a low temporal resolution, if present at all (Troccoli 2010). The MOS method thus depends on a suitable time averaging applied to the involved variables and inevitably operates at a low temporal resolution.

The S4ro.lm, S5ro.lm, and S5sro+ssro.lm benchmark models do not apply a time aggregation screening, but instead regress the predictand against the runoff simulations of the same 30-day time window. The results show that these benchmarks compete well against their counterparts (i.e., S4ro, S5ro, and S5sro+ssro; Fig. 2). Thus, for the predictors that carry the runoff simulations the additional effort of the time aggregation screening only leads to small improvements.

\section{2) LINEARITY}

The model formulation strictly assumes a linear relationship between the predictors and the predictand. From both an empirical as well as theoretical point of view, the assumption of linearity gains validity with an increasing time aggregation window length (Yuval and Hsieh 2002; Hsieh et al. 2003).
The residual analysis (not shown) reveals that low flows tend to be overpredicted and high flows tend to be underpredicted, often leading to skewed residual distributions. In addition, the pooled time series of the residuals sometimes exhibit autocorrelation. These issues could be related to missing predictors or imply that the time averaging windows of 20,30 , and 50 days are too short to completely linearize the predictor-predictand relationship.

However, the assumption of linearity is a technical constraint, too: Extrapolation beyond the domain covered by the training set leads to a few poor predictions, especially as some outliers are present in the S4 and S5 runoff simulations. For example, one of these outliers causes the large negative MSESS of the S5ro model in Fig. 4 for the Lower Bann. Subsequently, poor predictions become disastrous predictions when introducing interactions or higher-order terms due to overfitting or when transforming the predictand due to the necessary backtransform (not shown).

\section{b. Error model}

While the kernel density estimator is able to deal with skewed residual distributions, it otherwise assumes 
independent and identically distributed errors. The validation of the PIT values (Fig. 6) reveals some minor departures from uniformity. Given the model misspecifications reported above, the cross validation in combination with a rather small sample size, and the conservative significance level, we judge the reliability of the forecast probability distribution as reasonable.

However, the present MOS method uses the ensemble mean on the side of the predictors and thus ignores the ensemble spread-error relationship. This relationship is included in approaches such as the Bayesian model averaging (BMA) of Raftery et al. (2005) or the ensemble MOS (EMOS) of Gneiting et al. (2005).

The BMA or EMOS could be used in combination with the total runoff simulations analogously to the S4ro.lm and S5ro.lm benchmark models of the present study. Since the S4ro.lm and S5ro.lm benchmark models perform close to the level of the more complex model formulations, an application of the BMA and EMOS to the total runoff simulations could be worth an investigation.

\section{c. Predictor combinations}

Ignoring the trend and refRun models, the different predictor combinations arrive on average at a similar level of performance: The runoff-based models tend to slightly outperform the models containing precipitation and surface air temperature, which in turn tend to slightly outperform the persistence model (Figs. 2 and 3).

A notable exception that contrasts the different predictor combinations is provided by the Oder and the Neva River. For the Oder, the models based on meteorological predictors fail, but the runoff-based models score well, and vice versa for the Neva (Figs. 4 and 5). These two cases are briefly discussed now.

\section{1) The Oder CATCHMENT}

The Oder catchment differs from the other catchments particularly in two features:

1) According to the International Hydrogeological Map of Europe (IHME 2014), the lithology of the Oder catchment is dominated by coarse and fine sediments and the aquifer productivity is classified as low to moderate for nearly the entire catchment.

2) The runoff efficiency (streamflow divided by precipitation, equals for the Oder about 0.28 ) and total annual precipitation (about $500 \mathrm{~mm}$ ) belong to the lowest values contained in the present set of catchments.

The combination of high evapotranspiration and the presumably low contribution of groundwater from greater depths to streamflow might imply that the soil is the controlling factor for the generation of streamflow. If so, the model formulation based on the meteorological predictors is too simplistic to account for temporal variations of the soil moisture content.

\section{2) The Neva Catchment}

The preMet and refRun models score similar for the Neva catchment both at the 0-day as well as the 20-day lead time. In addition, the persistence model performs best among the tested predictor combinations (e.g., Fig. 5). This indicates that the initial hydrological conditions strongly control the generation of streamflow.

Besides its large catchment area, the Neva differs from the other catchments in the presence of several large lakes (e.g., Lake Ladoga, Lake Onega, and Lake Saimaa; see also Fig. 1). According to the Global Lakes and Wetlands Database (GLWD; Lehner and Döll 2004), about $14 \%\left(39000 \mathrm{~km}^{2}\right)$ of the catchment area is covered by lakes. Several of these lakes are regulated, for example, two dams regulate the Svir River, which connects Lake Onega with Lake Ladoga (Global Reservoir and Dam database version 1.1; Lehner et al. 2011).

While the S4 and S5 runoff simulations carry the information of the soil moisture content and snowpack at the date of prediction, the predictors based on preceding precipitation, temperature, or streamflow aim to account for the sum of all hydrological storages. Thus, we speculate that HTESSEL-based runoff is not a sufficient predictor if lakes represent a substantial fraction of the catchment area or if large artificial reservoirs are present.

To make the runoff-based models lake-aware one could experiment with additional predictors such as preceding precipitation and surface air temperature (similar to the preMet, S4PT, and S5PT models), lagged streamflow (as in the persistence model), or lake levels.

\section{d. Streamflow regulation}

As noted in section 2, the streamflow time series may contain numerous anthropogenic artifacts introduced by, for example, damming and regulation, water consumption, and diversions. While the temporal aggregation most likely cancels some of these anthropogenic signals, the potentially remaining human "noise" ends up in the predictand. Subsequently, it is theoretically possible that the MOS method learns anthropogenic patterns in the streamflow series.

A visual inspection of the daily streamflow series (not shown) reveals that obvious anthropogenic artifacts are 

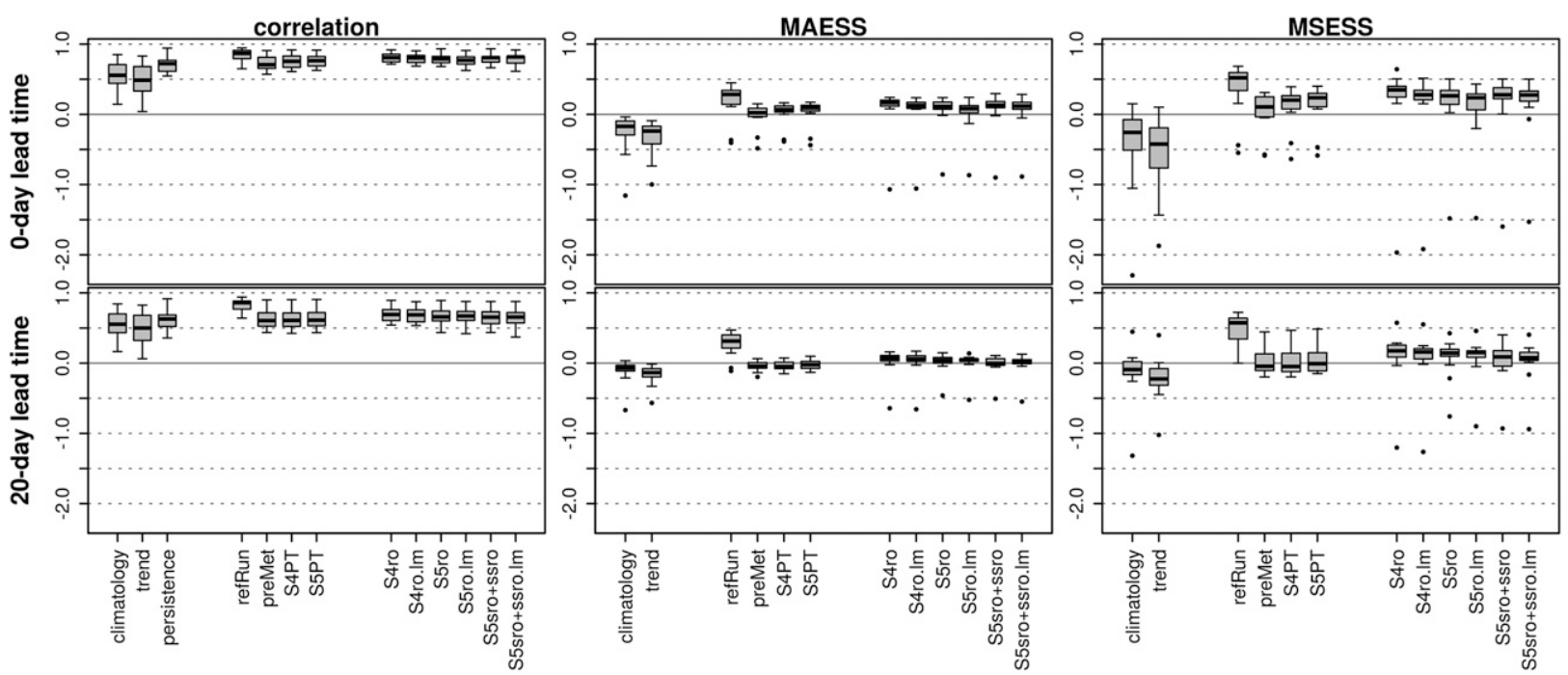

FIG. B1. Linear correlation, MAESS, and MSESS per model and catchment, based on the complete time series of predictions and observations. Shown are the (top) 0-day lead time and (bottom) 20-day lead time. The MAESS and MSESS are computed with respect to the streamflow persistence model; $n=16$.

mainly present for the Angerman, Glama, and Kemijoki Rivers. For these catchments the time series show some rectangular-like fluctuations at a frequency of a few days, most likely induced by streamflow regulation and hydro power production. However, the refRun model, which is aimed at estimating the potential skill, performs poorly mainly for the Duero and Oder River (Fig. 5). This indicates that human noise does not necessarily lead to a low forecast quality.

\section{e. Sources of skill}

Skill with respect to climatology restricts for most catchments and dates of prediction to the first month ahead (Figs. 2, 4, and 5). At the 20-day lead time skill is the exception; the high skill observed for the Neva might be enabled by the presence of several large lakes. In addition, the Neva seems to be the only catchment in which linear trends in the streamflow time series contribute to skill against climatology.

Furthermore, the results indicate that skill originates mostly from the initial hydrological conditions rather than from the predictions of precipitation and surface air temperature (e.g., the S5PT and S4PT models in Fig. 7). The initial conditions relevant for (sub)seasonal streamflow forecasting include hydrological storages such as soils, aquifers, surface water bodies, and snow (e.g., van Dijk et al. 2013; Shukla et al. 2013; Yossef et al. 2013).

The rather low contribution of ESM-simulated precipitation and surface air temperature to streamflow forecast is in line with recent studies: (sub)seasonal climate predictions show limited skill for the European domain. Besides the prediction of long-term trends, some skill is on average present within the first month ahead, but not beyond (Slater et al. 2017; Monhart et al. 2018; Rodrigues et al. 2018).

\section{f. Comparison with other studies}

Below, we select some studies with a hindcast configuration similar to the present study. Where appropriate we also compare numerical scores, however, it must be stressed that such a comparison bears some uncertainty due to the differences in the hindcast configurations.

Some of the following studies use the Ensemble Streamflow Prediction (ESP) framework (Wood and Lettenmaier 2008) for benchmarking. ESP model runs derive predictive skill exclusively from the initial hydrological conditions, what conceptually corresponds to the persistence and preMet models of the present study:

- Greuell et al. (2018) use the S4 hindcast archive in combination with the VIC model. For monthly mean streamflow forecasts validated against observations of about 700 European gauging stations, they report on average a correlation between 0.6 and 0.7 at the 0-day lead time. In the present study, the models using the S4 simulations score on average a correlation between 0.5 and 0.6 (Fig. 3).

- In Arnal et al. (2018) and Wetterhall and Di Giuseppe (2018), the LISFLOOD model is forced with the output from ECMWF's S4 and ENS-Extended systems in the European domain. In terms of the CRPSS, the ESP run 


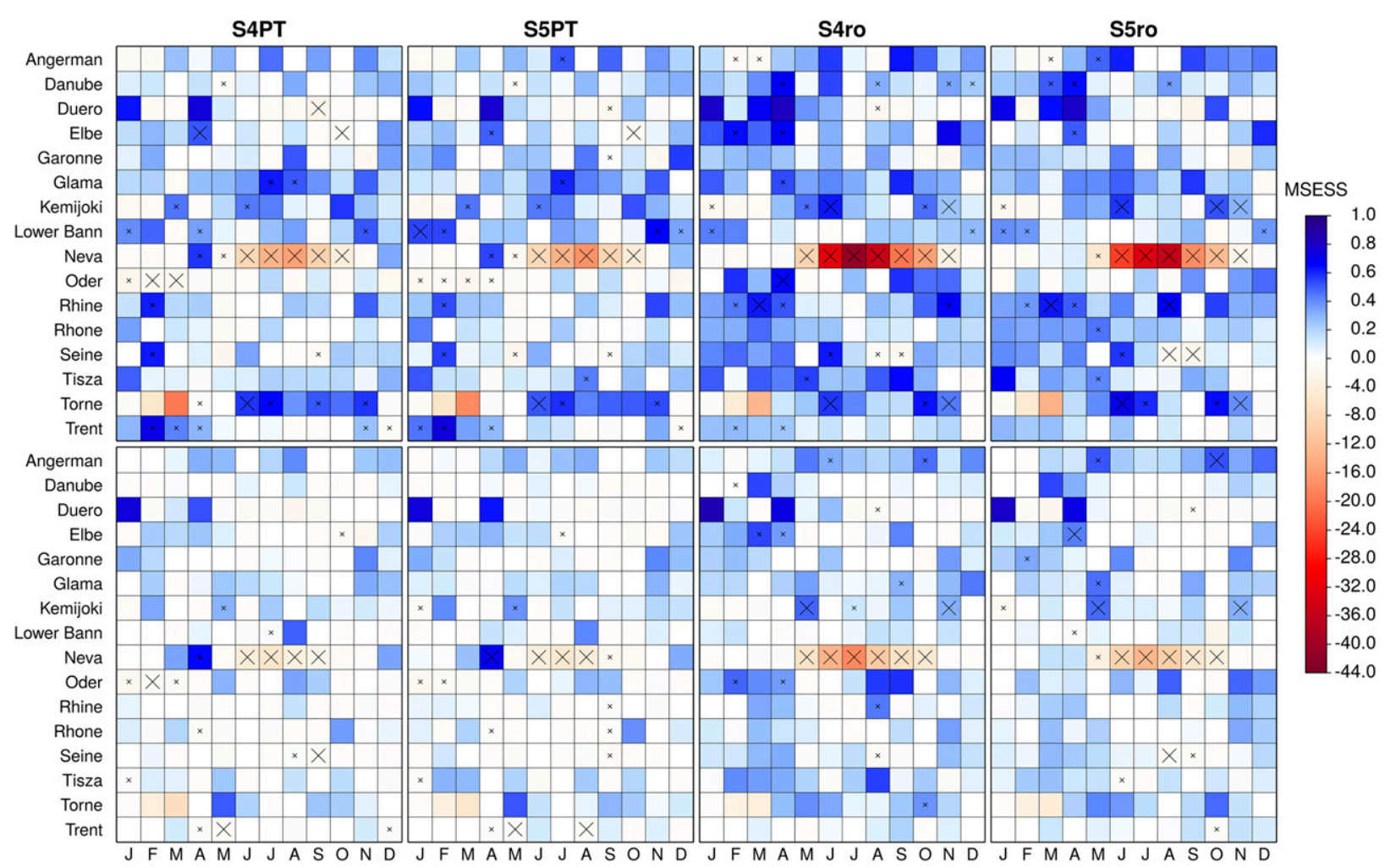

FIG. B2. MSESS at the (top) 0-day and (bottom) 20-day lead time with the streamflow persistence model as the benchmark. The months refer to the date of prediction. The $p$ values smaller than $0.01(0.05)$ for the null hypothesis "no difference in the MSE value" are indicated with a large (small) cross; $n=26$. Please note that the positive and negative parts of the color bar follow different gradients.

is outperformed on average within the first month, but not beyond. For monthly mean streamflow at the 0 -day lead time, the median CRPSS reaches in winter its maximum (Arnal et al. 2018). Thus, the present study agrees with the skillful lead time, but does not identify a skill peak in winter (Fig. 7).

- Monthly mean streamflow of the Elbe River at NeuDarchau is predicted in Meißner et al. (2017) with the LARSIM model and the S4 hindcast archive. At the 0day lead time, the MSESS with respect to climatology of the ESP run is for most months in the range of 0.4 to 0.7; for August, the MSESS is close to zero. Thus, both the magnitude and seasonal variations are approximately reproduced by the preMet model (Fig. 5). Benchmarking the LARSIM-S4 run with the ESP run in terms of the CRPS leads to a CRPSS of 0.16 in May and a CRPSS of 0.22 for June; otherwise the CRPSS stays close to zero at the 0-day lead time. In the present study, such high values for May and June are not reproduced (S4PT and S4ro models in Fig. 7).

In summary, the MOS method seems to reproduce several results of recent hindcast experiments, but tends to score smaller skill. Thus, MOS-processed ESM simulations could provide a benchmark for more complex (sub)seasonal streamflow forecast strategies to estimate "real" skill (Pappenberger et al. 2015).

\section{g. Pros and cons of the MOS method}

The MOS method features some generic advantages and disadvantages. Some of these are inherent to the data-driven approach, others are specific to the present prediction problem.

Advantages include:

- The ESM simulations do not need to be bias corrected.

- The predictor-predictand mapping might be able to bridge different spatial scales or to implicitly account for anthropogenic effects in the streamflow time series.

- Putting aside overfitted models, the MOS method should in principal fall back to climatology if the predictors are not correlated with the predictand (Zhao et al. 2017).

- Compared to forecast approaches that use the ESM output to force hydrological simulation models, the MOS method could save computational costs.

Disadvantages include:

- The temporal resolution of the predictand inevitably is low. 


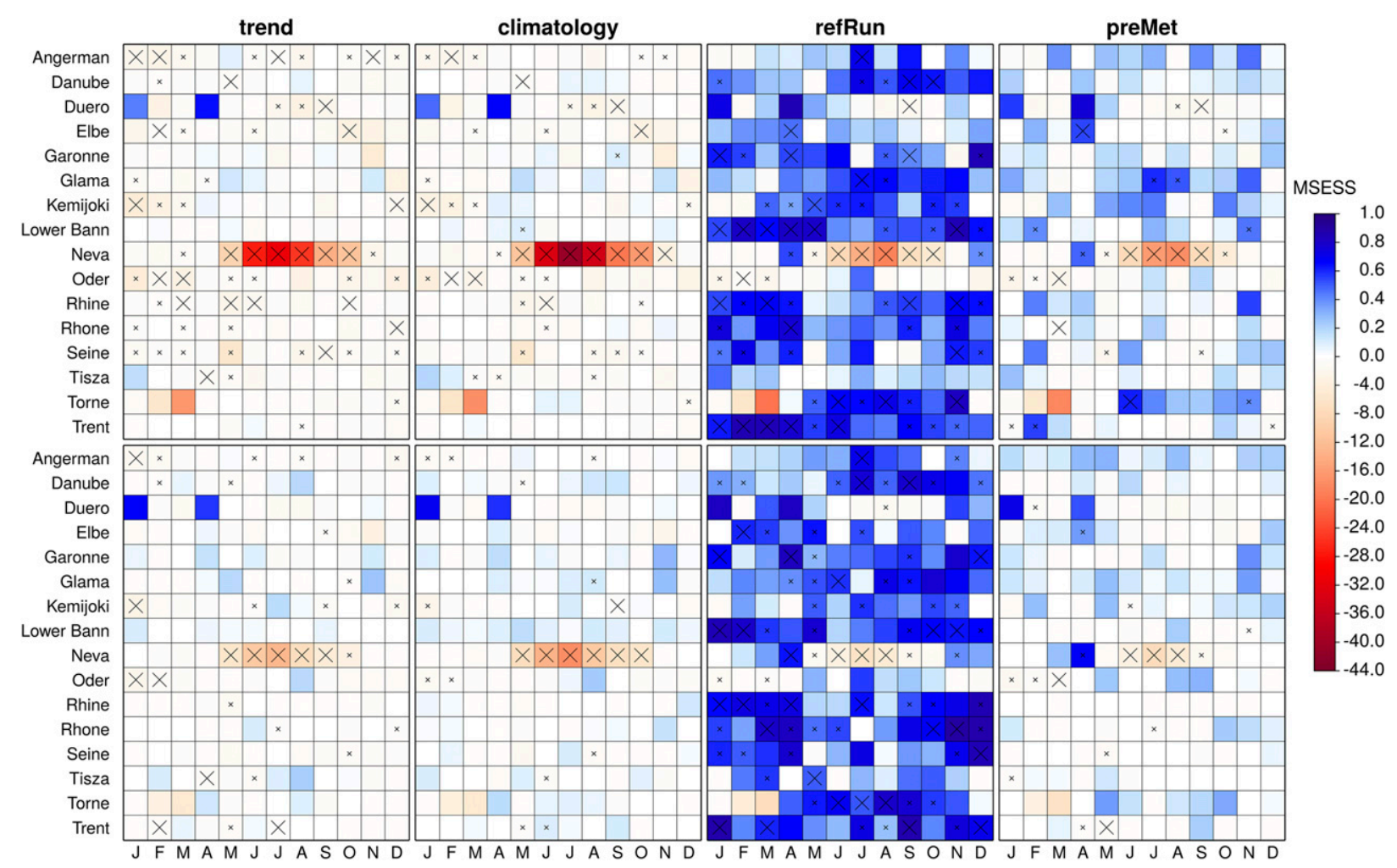

FIG. B3. MSESS at the (top) 0-day and (bottom) 20-day lead time with the streamflow persistence model as benchmark. The months refer to the date of prediction. The $p$ values smaller than $0.01(0.05)$ for the null hypothesis "no difference in the MSE value" are indicated with a large (small) cross; $n=26$. Please note that the positive and negative parts of the color bar follow different gradients.

- It is not feasible to visualize the forecast as an ensemble of hydrographs, which is often used by water managers.

- The method is data hungry, that is, the model fitting needs a sufficiently large training set, including past forecasts of the involved dynamical model. Consequently, it is impossible to rapidly integrate new observational data sources or to predict at locations along the river network where streamflow observations are not available.

\section{Conclusions}

Earth system models (ESMs) used today for subseasonal and seasonal forecasting of environmental conditions in general simulate runoff at the surface and at the bottom of the soil column. River streamflow, however, remains an unresolved variable and requires an additional modeling effort to forecast. The present study does so by an application of the model output statistics (MOS) method.

The test bed of the MOS application consists of 16 European catchments and monthly average streamflow at the 0-day and 20-day lead time in the period 1981-2006. Input to the MOS method is provided by the seasonal hindcast archive of the European Centre for MediumRange Weather Forecasts (ECMWF). Predictors are derived from both the S4 and SEAS5 forecast systems, namely surface runoff, subsurface runoff, total runoff, precipitation, and surface air temperature. In addition, the pool of candidate predictors contains observed precipitation and temperature preceding the date of prediction.

At the 0-day lead time the MOS method decreases the mean absolute error of the streamflow climatology by about $25 \%$ on average; at the 20 -day lead time, the decrease drops to about $9 \%$. This result holds for both the S4 and SEAS5 forecast systems. However, skill varies considerably between predictor combinations, catchments, and dates of prediction within the calendar year. In addition, skill is also frequently absent, especially at the 20-day lead time.

Benchmarking the MOS-processed ESM simulations with a streamflow persistence model further decreases skill. This holds in particular for a river system that features lakes, whose areas sum up to about $14 \%$ of the total catchment area. Aside from this catchment, the predictor combinations using the ESM runoff simulations tend to perform best on average.

Acknowledgments. We acknowledge the E-OBS data set from the EU-FP6 project ENSEMBLES (http:// ensembles-eu.metoffice.com/) and the data providers in 


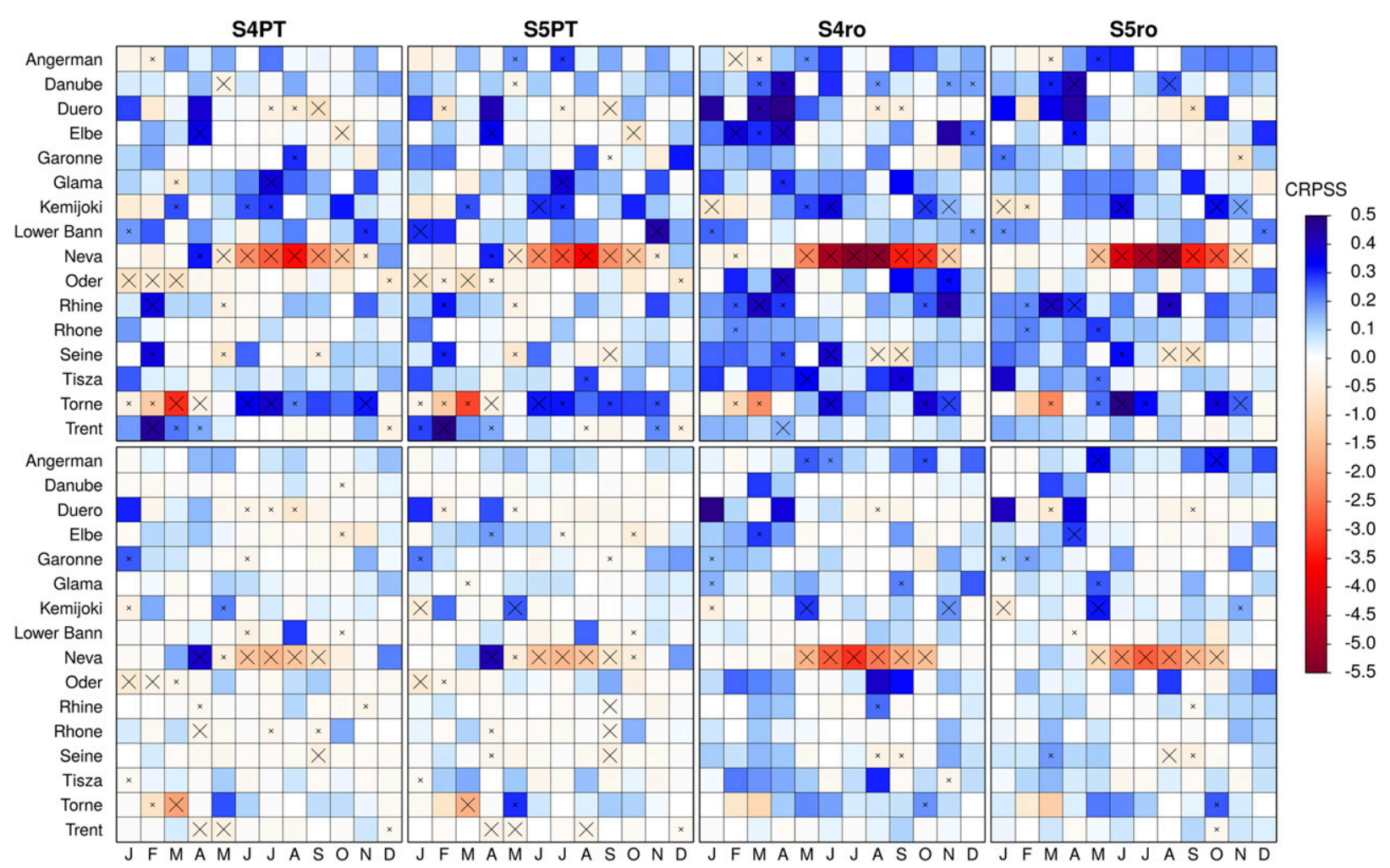

FIG. B4. CRPSS at the (top) 0-day and (bottom) 20-day lead time with the streamflow persistence model as the benchmark. The months refer to the date of prediction. The $p$ values smaller than $0.01(0.05)$ for the null hypothesis "no difference in the mean CRPS value" are indicated with a large (small) cross; $n=26$. Please note that the positive and negative parts of the color bar follow different gradients.

the ECA\&D project (www.ecad.eu) as well as the European Centre for Medium-Range Weather Forecasts for the access to its data archive. We also acknowledge the European Environmental Agency, the German Federal Institute for Geosciences and Natural Resources, the Natural Earth map data repository, and the World Wildlife Fund, who provided various geographical data. Streamflow observations were provided by the Global Runoff Data Centre, the French Ministry for an Ecological and Solidary Transition, and the Spanish Ministry of Agriculture and Fisheries, Food and Environment. Finally, we thank two anonymous reviewers for their valuable feedback that improved the manuscript substantially. The study was funded by the Group of Hydrology, which is part of the Institute of Geography at the University of Bern, Bern, Switzerland.

\section{APPENDIX A}

\section{Technical Note}

After aggregation in time, winter surface runoff (sro, used for the S5sro+ ssro model) can include years with zero and near-zero values as well as years with larger values. This is in particular the case for the Angerman, Kemijoki, and Torne catchments. Selecting in the bootstrap by chance only years with zero and near-zero values results in large regression coefficients and subsequently leads to disastrous overpredictions when applied to the out-of-sample cases.

As an empirical rule, we set all surface runoff values (after aggregation in time) smaller than $1 \mathrm{~m}^{3} \mathrm{~s}^{-1}$ to $0 \mathrm{~m}^{3} \mathrm{~s}^{-1}$. These $0 \mathrm{~m}^{3} \mathrm{~s}^{-1}$ surface runoff values frequently introduce singular covariance matrices. We set either of the regression coefficients of collinear variables to zero.

The regression approach from section $3 \mathrm{a}$ is implemented in an R package maintained on https://github.com/schiggo/ SSO.

\section{APPENDIX B}

\section{Additional Figures}

This appendix contains additional figures (Figs. B1-B4 ).

\section{REFERENCES}

Arnal, L., H. L. Cloke, E. Stephens, F. Wetterhall, C. Prudhomme, J. Neumann, B. Krzeminski, and F. Pappenberger, 2018: Skilful seasonal forecasts of streamflow over Europe? Hydrol. Earth Syst. Sci., 22, 2057-2072, https://doi.org/10.5194/hess-222057-2018. 
Balsamo, G., A. Beljaars, K. Scipal, P. Viterbo, B. van den Hurk, M. Hirschi, and A. K. Betts, 2009: A revised hydrology for the ECMWF model: Verification from field site to terrestrial water storage and impact in the Integrated Forecast System. J. Hydrometeor., 10, 623-643, https://doi.org/10.1175/ 2008JHM1068.1.

— , and Coauthors, 2015: ERA-Interim/Land: A global land surface reanalysis data set. Hydrol. Earth Syst. Sci., 19, 389407, https://doi.org/10.5194/hess-19-389-2015.

Barnston, A. G., and M. K. Tippett, 2017: Do statistical pattern corrections improve seasonal climate predictions in the North American Multimodel Ensemble models? J. Climate, 30, 8335-8355, https://doi.org/10.1175/JCLI-D-17-0054.1.

Breiman, L., 1996a: Bagging predictors. Mach. Learn., 24, 123-140, https://doi.org/10.1023/A:1018054314350.

_ 1996b: Out-of-bag estimation. University of California, 13 pp., https://www.stat.berkeley.edu/ breiman/OOBestimation.pdf.

Clark, M. P., and Coauthors, 2015: Improving the representation of hydrologic processes in Earth System Models. Water Resour. Res., 51, 5929-5956, https://doi.org/10.1002/2015WR017096.

D'Agostino, R. B., and M. A. Stephens, 1986: Goodness-of-Fit Techniques. Marcel Dekker, 576 pp.

E-OBS, 2017: Daily temperature and precipitation fields in Europe V.16. ECA\&D, http://www.ecad.eu/download/ensembles/ ensembles.php.

ECMWF, 2017: SEAS5 user guide. ECMWF, 43 pp., https:// www.ecmwf.int/sites/default/files/medialibrary/2017-10/System5_ guide.pdf.

—, 2018: IFS documentation. ECMWF, http://www.ecmwf.int/en/ forecasts/documentation-and-support/changes-ecmwf-model/ifsdocumentation.

ECRINS, 2012: European catchments and Rivers network system v1.1. EEA, http://www.eea.europa.eu/data-and-maps/data/ european-catchments-and-rivers-network.

Emerton, R., and Coauthors, 2018: Developing a global operational seasonal hydro-meteorological forecasting system: GloFAS-Seasonal v1.0. Geosci. Model Dev., 11, 3327-3346, https://doi.org/10.5194/gmd-11-3327-2018.

Foster, K. L., and C. B. Uvo, 2010: Seasonal streamflow forecast: A GCM multi-model downscaling approach. Hydrol. Res., 41, 503-507, https://doi.org/10.2166/nh.2010.143.

Glahn, H. R., and D. A. Lowry, 1972: The use of model output statistics (MOS) in objective weather forecasting. J. Appl. Meteor., 11, 1203-1211, https://doi.org/10.1175/1520-0450(1972) 011<1203:TUOMOS > 2.0.CO;2.

Gneiting, T., A. E. Raftery, A. H. Westveld III, and T. Goldman, 2005: Calibrated probabilistic forecasting using ensemble model output statistics and minimum crps estimation. Mon. Wea. Rev., 133, 1098-1118, https://doi.org/10.1175/MWR2904.1.

GRDC, 2016: The Global Runoff Data Centre. GRDC, http:// www.bafg.de/GRDC/EN/Home/homepage_node.html.

Greuell, W., W. H. P. Franssen, H. Biemans, and R. W. A. Hutjes, 2018: Seasonal streamflow forecasts for Europe - Part I: Hindcast verification with pseudo- and real observations. Hydrol. Earth Syst. Sci., 22, 3453-3472, https://doi.org/10.5194/ hess-22-3453-2018.

Haylock, M. R., N. Hofstra, A. M. G. Klein Tank, E. J. Klok, P. D. Jones, and M. New, 2008: A European daily high-resolution gridded data set of surface temperature and precipitation for 1950-2006. J. Geophys. Res., 113, D20119, https://doi.org/ 10.1029/2008JD010201.

Hersbach, H., 2000: Decomposition of the continuous ranked probability score for ensemble prediction systems. Wea.
Forecasting, 15, 559-570, https://doi.org/10.1175/1520-0434(2000) 015<0559:DOTCRP $>2.0 . C O ; 2$.

Hsieh, W. W., Yuval J. Li, A. Shabbar, and S. Smith, 2003: Seasonal prediction with error estimation of Columbia River streamflow in British Columbia. J. Water Res. Plann. Manage., 129, 146-149, https://doi.org/10.1061/(ASCE)0733-9496(2003)129: 2(146).

IHME, 2014: International Hydrogeological Map of Europe 1: 1,500,000 v1.1. IHME, https:/www.bgr.bund.de/EN/Themen/Wasser/ Projekte/laufend/Beratung//hme1500/ihme1500_projektbeschr_en.html.

Jones, M. C., J. S. Marron, and S. J. Sheather, 1996: A brief survey of bandwidth selection for density estimation. J. Amer. Stat. Assoc., 91, 401-407, https://doi.org/10.1080/01621459.1996.10476701.

Klein, W. H., and H. R. Glahn, 1974: Forecasting local weather by means of model output statistics. Bull. Amer. Meteor. Soc., 55 , 1217-1227, https://doi.org/10.1175/1520-0477(1974)055<1217: FLWBMO $>2.0 . \mathrm{CO} ; 2$.

Laio, F., and S. Tamea, 2007: Verification tools for probabilistic forecasts of continuous hydrological variables. Hydrol. Earth Syst. Sci., 11, 1267-1277, https://doi.org/10.5194/hess-11-12672007.

Landman, W. A., and L. Goddard, 2002: Statistical recalibration of GCM forecasts over southern Africa using model output statistics. J. Climate, 15, 2038-2055, https://doi.org/10.1175/15200442(2002)015<2038:SROGFO>2.0.CO;2.

Lehner, B., and P. Döll, 2004: Development and validation of a global database of lakes, reservoirs and wetlands. J. Hydrol., 296, 1-22, https://doi.org/10.1016/j.jhydrol.2004.03.028.

_ , and Coauthors, 2011: High-resolution mapping of the world's reservoirs and dams for sustainable river-flow management. Front. Ecol. Environ., 9, 494-502, https://doi.org/10.1890/100125.

Lehner, F., A. W. Wood, D. Llewellyn, D. B. Blatchford, A. G. Goodbody, and F. Pappenberger, 2017: Mitigating the impacts of climate nonstationarity on seasonal streamflow predictability in the U.S. Southwest. Geophys. Res. Lett., 44, 12 208-12 217, https://doi.org/10.1002/2017GL076043.

MAFFE, 2017: Spanish Ministry of Agriculture and Fisheries, Food and Environment. MAFFE, http://sig.mapama.es/redesseguimiento/visor.html?herramienta $=$ Aforos.

Meißner, D., B. Klein, and M. Ionita, 2017: Development of a monthly to seasonal forecast framework tailored to inland waterway transport in central Europe. Hydrol. Earth Syst. Sci., 21, 6401-6423, https://doi.org/10.5194/hess-21-6401-2017.

MEST, 2017: French Ministry for an Ecological and Solidary Transition. MEST, http://www.hydro.eaufrance.fr/.

Michaelsen, J., 1987: Cross-validation in statistical climate forecast models. J. Climate Appl. Meteor., 26, 1589-1600, https://doi.org/ 10.1175/1520-0450(1987)026<1589:CVISCF $>2.0 . C O ; 2$.

Monhart, S., C. Spirig, J. Bhend, K. Bogner, C. Schär, and M. A. Liniger, 2018: Skill of subseasonal forecasts in europe: effect of bias correction and downscaling using surface observations. J. Geophys. Res. Atmos., 123, 7999-8016, https://doi.org/ 10.1029/2017JD027923.

Mücher, C. A., J. A. Klijn, D. M. Wascher, and J. H. J. Schaminée, 2010: A new European Landscape Classification (LANMAP): A transparent, flexible and user-oriented methodology to distinguish landscapes. Ecol. Indic., 10, 87-103, https://doi.org/ 10.1016/j.ecolind.2009.03.018.

National Academies, 2016: Next Generation Earth System Prediction. 1st ed., National Academies Press, 350 pp., https:// doi.org/10.17226/21873.

Natural Earth, 2018: Free vector and raster map data. Natural Earth, http://www.naturalearthdata.com/. 
Nilsson, C., C. A. Reidy, M. Dynesius, and C. Revenga, 2005: Fragmentation and flow regulation of the world's large river systems. Science, 308, 405-408, https://doi.org/10.1126/ science.1107887.

Pappenberger, F., M. H. Ramos, H. L. Cloke, F. Wetterhall, L. Alfieri, K. Bogner, A. Mueller, and P. Salamon, 2015: How do I know if my forecasts are better? Using benchmarks in hydrological ensemble prediction. J. Hydrol., 522, 697-713, https://doi.org/10.1016/j.jhydrol.2015.01.024.

Peel, M. C., B. L. Finlayson, and T. A. McMahon, 2007: Updated world map of the Köppen-Geiger climate classification. Hydrol. Earth Syst. Sci., 11, 1633-1644, https://doi.org/10.5194/ hess-11-1633-2007.

R Core Team, 2018: R: A language and environment for statistical computing. R Foundation for Statistical Computing, https:// www.R-project.org/.

Raftery, A. E., T. Gneiting, F. Balabdaoui, and M. Polakowski, 2005: Using Bayesian model averaging to calibrate forecast ensembles. Mon. Wea. Rev., 133, 1155-1174, https://doi.org/ 10.1175/MWR2906.1.

Rodrigues, L. R. L., F. J. Doblas-Reyes, and C. A. S. Coelho, 2018: Calibration and combination of monthly near-surface temperature and precipitation predictions over Europe. Climate Dyn., https://doi.org/10.1007/s00382-018-4140-4.

Sahu, N., A. W. Robertson, R. Boer, S. Behera, D. G. DeWitt, K. Takara, M. Kumar, and R. B. Singh, 2017: Probabilistic seasonal streamflow forecasts of the Citarum River, Indonesia, based on general circulation models. Stochastic Environ. Res. Risk Assess., 31, 1747-1758, https://doi.org/10.1007/ s00477-016-1297-4.

Schefzik, R., T. L. Thorarinsdottir, and T. Gneiting, 2013: Uncertainty quantification in complex simulation models using ensemble copula coupling. Stat. Sci., 28, 616-640, https:// doi.org/10.1214/13-STS443.

Schick, S., O. Rössler, and R. Weingartner, 2016: Comparison of cross-validation and bootstrap aggregating for building a seasonal streamflow forecast model. Proc. IAHS, 374, 159-163.

,-- , and,- 2018 : Monthly streamflow forecasting at varying spatial scales in the Rhine basin. Hydrol. Earth Syst. Sci., 22, 929-942, https://doi.org/10.5194/hess-22-929-2018.

Sheather, S. J., and M. C. Jones, 1991: A reliable data-based bandwidth selection method for kernel density estimation. J. Roy. Stat. Soc. 53B, 683-690, http://www.jstor.org/stable/ 2345597.

Shukla, S., J. Sheffield, E. F. Wood, and D. P. Lettenmaier, 2013: On the sources of global land surface hydrologic predictability.
Hydrol. Earth Syst. Sci., 17, 2781-2796, https://doi.org/10.5194/ hess-17-2781-2013.

Slater, L. J., and G. Villarini, 2018: Enhancing the predictability of seasonal streamflow with a statistical-dynamical approach. Geophys. Res. Lett., 45, 6504-6513, https://doi.org/10.1029/ 2018 GL077945.

,-- , and A. A. Bradley, 2017: Weighting of NMME temperature and precipitation forecasts across Europe. J. Hydrol., 552, 646-659, https://doi.org/10.1016/j.jhydrol.2017.07.029.

Troccoli, A., 2010: Seasonal climate forecasting. Meteor. Appl., 17, 251-268, https://doi.org/10.1002/met.184.

van Dijk, A. I. J. M., J. L. Peña Arancibia, E. F. Wood, J. Sheffield, and H. E. Beck, 2013: Global analysis of seasonal streamflow predictability using an ensemble prediction system and observations from 6192 small catchments worldwide. Water Resour. Res., 49, 2729-2746, https://doi.org/10.1002/wrcr.20251.

Wetterhall, F., and F. Di Giuseppe, 2018: The benefit of seamless forecasts for hydrological predictions over Europe. Hydrol. Earth Syst. Sci., 22, 3409-3420, https://doi.org/10.5194/hess-223409-2018.

Wood, A. W., and D. P. Lettenmaier, 2008: An ensemble approach for attribution of hydrologic prediction uncertainty. Geophys. Res. Lett., 35, L14401, https://doi.org/10.1029/2008GL034648.

Yossef, N. C., H. Winsemius, A. Weerts, R. van Beek, and M. F. P. Bierkens, 2013: Skill of a global seasonal streamflow forecasting system, relative roles of initial conditions and meteorological forcing. Water Resour. Res., 49, 4687-4699, https:// doi.org/10.1002/wrcr.20350.

Yuan, X., and E. F. Wood, 2012: Downscaling precipitation or biascorrecting streamflow? Some implications for coupled general circulation model (CGCM)-based ensemble seasonal hydrologic forecast. Water Resour. Res., 48, W12519, https://doi.org/ 10.1029/2012WR012256.

- - — , and Z. Ma, 2015: A review on climate-model-based seasonal hydrologic forecasting: Physical understanding and system development. Wiley Interdiscip. Rev.: Water, 2, 523536, https://doi.org/10.1002/wat2.1088.

Yuval, and W. W. Hsieh, 2002: The impact of time-averaging on the detectability of nonlinear empirical relations. Quart. J. Roy. Meteor. Soc., 128, 1609-1622, https://doi.org/10.1002/ qj.200212858311.

Zhao, T., J. C. Bennett, Q. J. Wang, A. Schepen, A. W. Wood, D. E. Robertson, and M.-H. Ramos, 2017: How suitable is quantile mapping for postprocessing GCM precipitation forecasts? J. Climate, 30, 3185-3196, https://doi.org/10.1175/JCLI-D-160652.1. 